UDC 519.1

\title{
A Note on Hamiltonian Bypasses in Digraphs with Large Degrees
}

\author{
Samvel Kh. Darbinyan \\ Institute for Informatics and Automation Problems of NAS RA \\ e-mail: samdarbin@iiap.am
}

\begin{abstract}
Let $D$ be a 2 -strongly connected directed graph of order $p \geq 3$. Suppose that $d(x) \geq p$ for every vertex $x \in V(D) \backslash\left\{x_{0}\right\}$, where $x_{0}$ is a vertex of $D$. In this paper, we show that if $D$ is Hamiltonian or $d\left(x_{0}\right)>2(p-1) / 5$, then $D$ contains a Hamiltonian path, in which the initial vertex dominates the terminal vertex.
\end{abstract}

Keywords: Digraph, cycle, Hamiltonian cycle, Hamiltonian bypass.

\section{Introduction}

In this paper, we consider finite digraphs (directed graphs) without loops and multiple arcs. Every cycle and path are assumed to be simple and directed. We shall assume that the reader is familiar with the standard terminology on digraphs and refer the reader to [1]. A cycle (path) in a digraph $D$ passing through all the vertices of $D$ is called Hamiltonian. A digraph containing a Hamiltonian cycle is called a Hamiltonian digraph. A Hamiltonian path in a digraph $D$ in which the initial vertex dominates the terminal vertex is called a Hamiltonian bypass. There are numerous sufficient conditions for the existence of a Hamiltonian cycle in digraphs (see, e.g., $[1,2,3]$ ). It is natural to consider an analogous problem for the existence of a Hamiltonian bypass.

It was proved in [4] - [9] that a number of sufficient conditions for a digraph to be Hamiltonian is also sufficient for a digraph to contain a Hamiltonian bypass (with some exceptions which are characterized). In particular, Theorems 1.4 and 1.5 were proved in [5] and [6], respectively. To formulate these theorems, we need the following definitions.

Definition 1: Let $D_{0}$ denote any digraph of order $p \geq 3$, $p$ is odd, such that $V\left(D_{0}\right)=A \cup B$, where $A \cap B=\emptyset, A$ is an independent set with $(p+1) / 2$ vertices, $B$ is a set of $(p-1) / 2$ vertices inducing an arbitrary subdigraph, and $D_{0}$ contains all the possible arcs between $A$ and $B$.

Definition 2: For any $k \in[1, p-2]$ let $D_{p-k, k}$ denote a digraph of order $p \geq 3$, obtained from $K_{p-k}^{*}$ and $K_{k+1}^{*}$ by identifying a vertex of the first with a vertex of the second. 
Definition 3: By $T_{5}$ we denote a tournament of order 5 with vertex set $\left\{x_{1}, x_{2}, x_{3}, x_{4}, y\right\}$ and arc set $\left\{x_{i} x_{i+1} \mid i \in[1,3]\right\} \cup\left\{x_{4} x_{1}, x_{1} y, x_{3} y, y x_{2}, y x_{4}, x_{1} x_{3}, x_{2} x_{4}\right\}$.

Theorem 1: (Benhocine [5]). Let D be a 2-strong digraph of order $p$ with minimum degree at least $p-1$. Then $D$ contains a Hamiltonian bypass, unless $D$ is isomorphic to a digraph of type $D_{0}$.

Theorem 2: (Darbinyan [6]). Let $D$ be a strong digraph of order $p \geq 3$. Suppose that $d(x)+d(y) \geq 2 p-2$ for every pair of non-adjacent vertices $x$, $y$ of $V(D)$. Then $D$ contains a Hamiltonian bypass, unless $D$ is isomorphic to a digraph of the set $D_{0} \cup\left\{D_{p-k, k}, T_{5}, C_{3}\right\}$.

The author [10] proved the following results.

Theorem 3: (Darbinyan [10]). For every integer $p \geq 8$ there is a 2-strong non-Hamiltonian digraph of order $p$, which has $p-1$ vertices of degrees at least $p$.

Theorem 4: (Darbinyan [9]). Let D be a 2-strong digraph of order $p \geq 3$ with the minimum degree at least $p-4$. If $p-1$ vertices of $D$ have degrees at least $p$, then $D$ is Hamiltonian.

Theorem 5: (Darbinyan [10]). Let $D$ be a strong digraph of order $p \geq 3$. Suppose that $d(x)+d(y) \geq 2 p-1$ for every pair of non-adjacent vertices $x, y \in V(D) \backslash\left\{z_{0}\right\}$, where $z_{0}$ is some vertex in $V(D)$. Then $D$ contains a cycle of length at least $p-1$.

The following corollary follows from Theorem 5 .

Corollary 1: Let $D$ be a strong digraph of order $p \geq 3$. If $p-1$ vertices of $V(D)$ have degrees at least $p$, then $D$ is Hamiltonian or contains a cycle of length $p-1$ (in fact, $D$ has a cycle that contains all the vertices with degrees at least $p$ ).

Remark 1: For the proof of Theorem 3, it suffices to consider a digraph $H(n)$ of order $n \geq 8$, which is defined as follows:

$$
\begin{gathered}
V(H(n)):=\left\{x_{0}, x_{1}, x_{2}, \ldots, x_{n-4}, y_{1}, y_{2}, y_{3}\right\} \text { and } \\
A(H(n)):=\left\{y_{i} y_{j} \mid i \neq j\right\} \cup\left\{x_{i} x_{i+1} \mid 0 \leq i \leq n-4\right\} \cup\left\{y_{i} x_{j} \mid 1 \leq i \leq 3,1 \leq j \leq n-6\right\} \\
\cup\left\{x_{i} x_{j} \mid 1 \leq j<i \leq n-4\right\} \cup\left\{x_{n-4} y_{i}, x_{n-6} \mid 1 \leq i \leq 3\right\} \cup\left\{x_{i} x_{n-5} \mid 1 \leq i \leq n-7\right\} \\
\cup\left\{x_{0} x_{n-5}, x_{n-5} x_{0}, x_{n-4} x_{0}, x_{n-6} x_{n-4}\right\} .
\end{gathered}
$$

Note that Theorem 3 disproves a conjecture of Thomassen ([2]. Every 3-strong digraph of order $p$ with minimum degree at least $p+1$ is strongly Hamitonian-connected).

In this paper, we prove the following theorem.

Theorem 6: Let $D$ be a 2-strong digraph of order $p \geq 3$. Suppose that $d(x) \geq p$ for every vertex $x \in V(D) \backslash\left\{x_{0}\right\}$, where $x_{0}$ is a vertex of $D$. If $D$ is Hamiltonian or $d\left(x_{0}\right)>2(p-1) / 5$, then $D$ contains a Hamiltonian bypass. 


\section{Terminology and Notation}

In this paper, we consider finite digraphs without loops and multiple arcs. For a digraph $D$, we denote by $V(D)$ the vertex set of $D$ and by $A(D)$ the set of $\operatorname{arcs}$ in $D$. The order of $D$ is the number of its vertices. Let $x, y$ be distinct vertices in $D$. The arc of a digraph $D$ directed from $x$ to $y$ is denoted by $x y$ (we say that $x$ dominates $y$ ). For disjoint subsets $A$ and $B$ of $V(D)$ we define $A(A \rightarrow B)$ as the set $\{x y \in E(D) \mid x \in A, y \in B\}, A(A, B)=A(A \rightarrow B) \cup A(B \rightarrow A)$. The notation $A \rightarrow B$ denotes that every vertex of $A$ dominates every vertex of $B$. $A \mapsto B$ means that $A \rightarrow B$ and there is no arc from a vertex of $B$ to a vertex of $A$. If $x \in V(D)$ and $A=\{x\}$, we write $x$ instead of $\{x\}$. The out-neighborhood of a vertex $x$ is the set $N^{+}(x)=$ $\{y \in V(D) \mid x y \in A(D)\}$ and $N^{-}(x)=\{y \in V(D) \mid y x \in A(D)\}$ is the in-neighborhood of $x$. Similarly, if $A \subseteq V(D)$, then $N^{+}(x, A)=\{y \in A \mid x y \in A(D)\}$ and $N^{-}(x, A)=\{y \in$ $A \mid y x \in A(D)\}$. The out-degree of $x$ is $d^{+}(x)=\left|N^{+}(x)\right|$ and $d^{-}(x)=\left|N^{-}(x)\right|$ is the in-degree of $x$. Similarly, $d^{+}(x, A)=\left|N^{+}(x, A)\right|$ and $d^{-}(x, A)=\left|N^{-}(x, A)\right|$. The degree of the vertex $x$ in $D$ is defined as $d(x)=d^{+}(x)+d^{-}(x)$ (similarly, $d(x, A)=d^{+}(x, A)+d^{-}(x, A)$ ).

The subdigraph of $D$ induced by a subset $A$ of $V(D)$ is denoted by $D[A]$. The path (respectively, the cycle) consisting of the distinct vertices $x_{1}, x_{2}, \ldots, x_{m}(m \geq 2)$ and the $\operatorname{arcs} x_{i} x_{i+1}, 1 \leq i \leq m-1$ (respectively, $x_{i} x_{i+1}, 1 \leq i \leq m-1$, and $x_{m} x_{1}$ ), is denoted by $x_{1} x_{2} \cdots x_{m}$ (respectively, $x_{1} x_{2} \cdots x_{m} x_{1}$ ). We say that $x_{1} x_{2} \cdots x_{m}$ is a path from $x_{1}$ to $x_{m}$ or is an $\left(x_{1}, x_{m}\right)$-path. The length of a cycle or a path is the number of its arcs. A cycle of length $k, k \geq 2$, is denoted by $C_{k}$. For a cycle $C_{k}:=x_{1} x_{2} \cdots x_{k} x_{1}$, the subscripts considered modulo $k$, i.e., $x_{i}=x_{s}$ for every $s$ and $i$ such that $i \equiv s(\bmod k)$. If $P$ is a path containing a subpath from $x$ to $y$, we let $P[x, y]$ denote that subpath. Similarly, if $C$ is a cycle containing vertices $x$ and $y, C[x, y]$ denotes the subpath of $C$ from $x$ to $y$. For a digraph $D$ of order $n$, by $D(n, 2)=\left[x_{1} x_{n} ; x_{1} x_{2} x_{3} \ldots x_{n}\right]$ we denote a Hamiltonian path in which the initial vertex $x_{1}$ dominates the terminal vertex $x_{n}$.

A digraph $D$ is strongly connected (or, just, strong) if there exists a path from $x$ to $y$ and a path from $y$ to $x$ for every pair of distinct vertices $x, y$. A digraph $D$ is $k$-strongly connected (or, $k$-strong), if $|V(D)| \geq k+1$ and $D[V(D) \backslash A]$ is strong for any set $A$ of at most $k-1$ vertices. Two distinct vertices $x$ and $y$ of a digraph $D$ are adjacent if $x y \in A(D)$ or $y x \in A(D)$ (or both). By $K_{n}^{*}$ is denoted the complete digraph of order $n$.

\section{Preliminaries}

The following well-known simple Lemmas 1-3 are the basis of our results and other theorems on directed cycles and paths in digraphs. They will be used extensively in the proof of our result.

Lemma 1: (Häggkvist and Thomassen [12]). Let $D$ be a digraph of order $p \geq 3$ containing a cycle $C_{m}, 2 \leq m \leq p-1$. Let $x$ be a vertex not contained in this cycle. If $d\left(x, V\left(C_{m}\right)\right) \geq m+1$, then for every $k, 2 \leq k \leq m+1, D$ contains a cycle of length $k$ including $x$.

The following lemma is a modification of a lemma by Bondy and Thomassen [13].

Lemma 2: Let $D$ be a digraph of order $p \geq 3$ containing a path $P:=x_{1} x_{2} \ldots x_{m}, 2 \leq m \leq$ $p-1$ and $x$ be a vertex not contained in this path. If one of the following conditions holds: 
(i) $d(x, V(P)) \geq m+2$;

(ii) $d(x, V(P)) \geq m+1$ and $x x_{1} \notin A(D)$ or $x_{m} x \notin A(D)$;

(iii) $d(x, V(P)) \geq m, x x_{1} \notin A(D)$ and $x_{m} x \notin A(D)$;

then there is an $i, 1 \leq i \leq m-1$, such that $x_{i} x, x x_{i+1} \in A(D)$ i.e., $x_{1} x_{2} \ldots x_{i} x x_{i+1} \ldots x_{m}$ is a path of length $m D$ (we say that $x$ can be inserted into $P$ ).

The following lemma is a simple extension of a lemma by Bang-Jensen, Gutin and Li [14].

Lemma 3: Let $P=u_{1} u_{2} \ldots u_{s}$ be a path in a digraph $D$ (possibly, $s=1$ ) and let $Q=v_{1} v_{2} \ldots v_{t}$ be a path (or $Q=v_{1} v_{2} \ldots v_{t} v_{1}$ be a cycle) in $D[V(D) \backslash V(Q)], t \geq 2$. Suppose that for each $u_{i}, 1 \leq i \leq s$, there is an arc $v_{j} v_{j+1}$ on $Q$ such that $v_{j} u_{i}, u_{i} v_{j+1} \in A(D)$. Then there is a $\left(v_{1}, v_{t}\right)$-path (or a cycle) of length $t+k-1$ (respectively, $\left.t+k\right), 1 \leq k \leq s$, with vertex set $\left\{v_{1}, v_{2}, \ldots, v_{t}\right\} \cup\left\{u_{1}, u_{2}, \ldots, u_{k}\right\}$.

\section{Proofs of the Main Results}

Theorem 6: Let $D$ be a 2-strong digraph of order $p \geq 3$. Suppose that $d(x) \geq p$ for every vertex $x \in V(D) \backslash\left\{x_{0}\right\}$, where $x_{0}$ is a vertex of $D$. If $D$ is Hamiltonian or $d\left(x_{0}\right)>2(p-1) / 5$, then $D$ contains a Hamiltonian bypass.

Proof: Suppose, on the contrary, that is $D$ contains no Hamiltonian bypass. We first will prove the following claim (note that in the proofs of Claim 1 and Case 1, we do not use the fact that $D$ is 2 -strong).

Claim 1: $D$ has no cycle of length $l$ through $x_{0}$, where $l=p-1$ or $l=p-2$.

Proof: Suppose that the claim is not true. Assume that $C_{p-1}=x_{1} x_{2} \ldots$

$x_{p-1} x_{1}, x_{0} \in V\left(C_{p-1}\right)$ and $y \notin V\left(C_{p-1}\right)$. Since $D$ contains no Hamiltonian bypass, for every $i, 1 \leq i \leq p-1$, we have $d^{+}\left(y,\left\{x_{i}, x_{i+1}\right\}\right) \leq 1$ and $d^{-}\left(y,\left\{x_{i}, x_{i+1}\right\}\right) \leq 1$. Therefore,

$$
2 d(y)=\sum_{i=1}^{p-1}\left(d^{+}\left(y,\left\{x_{i}, x_{i+1}\right\}\right)+d^{-}\left(y,\left\{x_{i}, x_{i+1}\right\}\right)\right) \leq 2(p-1),
$$

which contradicts that $d(y) \geq p$. Thus, $D$ contains no cycle of length $p-1$ through $x_{0}$.

Now assume that $D$ contains a cycle of length $p-2$ through $x_{0}$. Let $C_{p-2}=x_{1} x_{2} \ldots$ $x_{p-2} x_{1}, x_{0} \in V\left(C_{p-2}\right)$ and $x, y \notin V\left(C_{p-2}\right)$. Since $D$ contains no cycle of length $p-1$ through $x_{0}$, from Lemma 1 it follows that $x y, y x \in A(D), d\left(x, V\left(C_{p-2}\right)\right)=d\left(y, V\left(C_{p-2}\right)\right)=$ $p-2$ and there is a vertex $x_{i}$ such that the vertices $x, x_{i}$ are not adjacent and the arcs $x_{i-1} x, x x_{i+1}$ are in $D$. If $y x_{i} \in A(D)$, then $D(p, 2)=\left[y x_{i} ; y x C\left[x_{i+1}, x_{i}\right]\right]$, if $x_{i} y \in A(D)$, then $D(p, 2)=\left[x_{i} y ; C\left[x_{i}, x_{i-1}\right] x y\right]$, a contradiction. We may therefore assume that $x_{i}$ and $y$ also are not adjacent. Using this, Lemmas 1, 2 and the fact that $D$ contains no cycle of length $p-1$ througt $x_{0}$, we obtain that $x_{i}=x_{0}, x_{i-1} y, y x_{i+1} \in A(D)$ and the vertex $x$ $(y)$ is adjacent to every vertex in $V(D) \backslash\left\{x_{0}\right\}$. Hence, we have that if $x x_{i+2} \in A(D)$, then $D(p, 2)=\left[y x_{i+1} ; y x C\left[x_{i+2}, x_{i+1}\right]\right]$, a contradiction. If $x x_{i+2} \notin A(D)$, then $x_{i-2} x \in A(D)$ and $D(p, 2)=\left[x_{i-1} y ; C\left[x_{i-1}, x_{i-2}\right] x y\right]$, a contradiction. Claim 1 is proved.

Now, we divide the proof into two cases to consider.

Case 1. $D$ is Hamiltonian. 
Let $C_{p}=x_{1} x_{2} \ldots x_{p} x_{1}$ be a Hamiltonian cycle in $D$. Since $D$ contains no Hamiltonian bypass, we have that $x_{i+1} x_{i} \notin A(D)$ for every $i, 1 \leq i \leq p$. Using this, it is not difficult to check that if $p \leq 6$, then $D$ contains a Hamiltonian bypass. We may therefore assume that $p \geq 7$.

Claim 2: If $x_{0} \neq x_{i+1}$, then the vertices $x_{i}$ and $x_{i+2}$ are not adjasent, where $1 \leq i \leq p$.

Proof: Suppose, on the contrary, that is for some $i, 1 \leq i \leq p, x_{0} \neq x_{i+1}$ and the vertices $x_{i}, x_{i+2}$ are adjasent. Without loss of generality we may assume that $i=1$. Since $x_{0} \neq x_{2}$, we have $d\left(x_{2}\right) \geq p$ and, by Claim $1, x_{1} x_{3} \notin A(D)$. Hence, $x_{3} x_{1} \in A(D)$. It is clear that $x_{0} \neq x_{1}$ or $x_{0} \neq x_{3}$. This together with Claim 1 implies that $x_{p} x_{2} \notin A(D)$ or $x_{2} x_{4} \notin A(D)$. Since there is no $\left(x_{3}, x_{1}\right)$-Hamiltonian path, using Lemma 2(ii), we obtain that

$$
d\left(x_{2}, V(D) \backslash\left\{x_{2}\right\}\right)=d\left(x_{2},\left\{x_{1}, x_{3}\right\}\right)+d\left(x_{2}, V(D) \backslash\left\{x_{1}, x_{2}, x_{3}\right\}\right) \leq 2+p-3=p-1,
$$

which contradicts that $d\left(x_{2}\right) \geq p$.

It is not difficult to show that there are two distinct vertices $x_{i}$ and $x_{i+k}$ such that $x_{i+k} x_{i} \in A(D)$ and $x_{0} \notin\left\{x_{i+1}, x_{i+2}, \ldots, x_{i+k-1}\right\}$. We may assume that $k$ is chosen so that $k$ is the smallest possible. Without loss of generality we may assume that $i=1$. Then $d^{-}\left(x_{1},\left\{x_{2}, x_{3}, \ldots, x_{k}\right\}\right)=0$. From Claim 2 it follows that $3 \leq k \leq p-2$.

Assume first $k=3$, i.e., $x_{4} x_{1} \in A(D)$. By Claim 2, the vertices $x_{2}$ and $x_{4}\left(x_{1}\right.$ and $\left.x_{3}\right)$ are not adjacent since $x_{0} \notin\left\{x_{2}, x_{3}\right\}$. Now from $x_{i+1} x_{i} \notin A(D), d\left(x_{2}\right) \geq p$ and $d\left(x_{3}\right) \geq p$ it follows that

$$
d\left(x_{2},\left\{x_{5}, x_{6}, \ldots, x_{p}\right\}\right) \geq p-2 \quad \text { and } \quad d\left(x_{3},\left\{x_{5}, x_{6}, \ldots, x_{p}\right\}\right) \geq p-2 .
$$

Hence, by Lemma 2 , the vertex $x_{2}\left(x_{3}\right)$ can be inserted into $x_{5} x_{6} \ldots x_{p}$. Then, by Lemma 3 , there is an $\left(x_{4}, x_{1}\right)$-Hamiltonian path, which is a contradiction as $x_{4} x_{1} \in A(D)$.

Assume next that $k \geq 4$. By Claim 2, the vertices $x_{i}$ and $x_{i+2}$, where $1 \leq i \leq k-1$ are not adjacent. From the minimality of $k$ it follows that if $1 \leq i<j \leq k+1$, then $x_{j} x_{i} \in A(D)$ if and only if $j=k+1$ and $i=1$. From the minimality of $k \geq 4$ and Claim 1 it follows that for each $x_{i} \in\left\{x_{1}, x_{2}, \ldots, x_{k-2}\right\}$,

$$
d\left(x_{i},\left\{x_{i+2}, x_{i+3}\right\}\right)=d\left(x_{k-1},\left\{x_{k+1}\right\}\right)=0 .
$$

Also we need to show the following claim.

Claim 3: Suppose that $1 \leq i<j-1 \leq k$. Then $x_{i} x_{j} \in A(D)$ if and only if $i=1$ and $j=k+1$.

Proof: For a proof by contradiction, suppose that $x_{m} x_{n} \in A(D)$, where $1 \leq m<n-1 \leq k$ and $m \neq 1$ or $n \neq k+1$. Without loss of generality, we may assume that $n-m$ is the minimum possible. From (1) it follows that $n-m \geq 4$, i.e., $\left|\left\{x_{m+1}, \ldots, x_{n-1}\right\}\right|=n-m-1 \geq 3$. Note that $R:=x_{m} x_{n} x_{n+1} \ldots x_{p} x_{1} x_{2} \ldots x_{m}$ is a cycle of length $p-n+m+1 \leq p-3$ through $x_{0}$. By the minimality of $k$ and $n-m$, for every $y \in\left\{x_{m+1}, \ldots, x_{n-1}\right\}$ we have

$$
d\left(y,\left\{x_{m+1}, \ldots, x_{n-1}\right\}\right) \leq 2 \text { and } d(y, V(R)) \geq p-2 .
$$

Therefore, by Lemma 1 , every vertex $y \in\left\{x_{m+1}, \ldots, x_{n-1}\right\}$ can be inserted into $R$. Now using Lemma 3 , we obtain a cycle of length $p-1$ through $x_{0}$, which contradicts Claim 1 .

From Claim 3 and the minimality of $k \geq 4$ it follows that

$$
d\left(x_{2},\left\{x_{2}, x_{3}, \ldots, x_{k}\right\}\right)=d\left(x_{k},\left\{x_{2}, x_{3}, \ldots, x_{k}\right\}\right)=1
$$


and for every $i, 3 \leq i \leq k-1, d\left(x_{i},\left\{x_{2}, x_{3}, \ldots, x_{k}\right\}\right)=2$. Therefore, $d\left(x_{i}, V(Q)\right) \geq p-2$, where $2 \leq i \leq k$ and $Q:=x_{k+1} x_{k+2} \ldots x_{p} x_{1}$. Note that $|V(Q)|=p-k+1$. If $k \geq 5$, then $|V(Q)| \leq p-4$, and, by Lemma $2(\mathrm{i})$, every vertex $x_{i}, 2 \leq i \leq k$, can be inserted into $Q$. If $k=4$, then $|V(Q)|=p-3, d\left(x_{2}, V(Q)\right) \geq p-1, d\left(x_{4}, V(Q)\right) \geq p-1$ and $d\left(x_{3}, V(Q)\right) \geq p-2$. Since $d\left(x_{3},\left\{x_{1}, x_{5}\right\}\right)=0$, again using Lemma 2 , we obtain that each vertex $x_{i} \in\left\{x_{2}, x_{3}, x_{4}\right\}$ can be inserted into $Q$. Therefore, by Lemma 3 , there is an $\left(x_{k+1}, x_{1}\right)$-Hamiltonian path, which contradicts our initial supposition since $x_{k} x_{1} \in A(D)$. The discussion of Case 1 is completed.

Case 2. $D$ is not Hamiltonian.

(*) Observe that by Claime 1 , in this case every cycle through $x_{0}$ in $D$ has length at most $p-3$.

Then, by Corollary $1, D$ contains a cycle of length $p-1$. Let $C_{p-1}=x_{1} x_{2} \ldots x_{p-1} x_{1}$ be a cycle of length $p-1$ in $D$. By Claim 1, $x_{0} \in V\left(C_{p-1}\right)$. For this case, we first give the following claim and lemma.

Claim 4: Let $P:=x_{1} x_{2} \ldots x_{p-1}$ be an $\left(x_{1}, x_{p-1}\right)$-path of length $p-2$ through $x_{0}$ in $D$. Then $x_{1} x_{p-1} \notin A(D)$.

Proof: For a proof by contradiction, suppose that $x_{1} x_{p-1} \in A(D)$. Let $x \notin V(P)$. Then $d(x) \geq p$ since $x \neq x_{0}$. Since $D$ contains no Hamiltonian bypass, it follows that $x$ cannot be inserted into $P$. Now using Lemma $2(\mathrm{i})$ and $d(x) \geq p$, we obtain that $x_{p-1} x$ and $x x_{1} \in A(D)$. Therefore, $x_{1} x_{2} \ldots x_{p-1} x x_{1}$ is a Hamiltonian cycle in $D$, which contradicts the hypothesis of this case.

Lemma 4: $D$ contains no cycle of length $p-3$ through $x_{0}$.

Proof: Suppose that the lemma is not true. Let $C:=x_{1} x_{2} \ldots$

$x_{p-4} x_{0} x_{1}$ be a cycle of length $p-3$ through $x_{0}$ in $D$ and let $B:=V(D) \backslash V(C)$. By Claim $1, D$ contains no cycle of length $p-1$ and $p-2$ through $x_{0}$. This together with Lemma 1 implies that for every $y \in B$,

$$
p \leq d(y)=d(y, V(C))+d(y, B) \leq p-3+d(y, B) .
$$

Therefore, $d(y, B) \geq 3$. This implies that $D[B]$ is Hamiltonian since $|B|=3$, in particular, $D[B]$ is strong.

We now consider the folowing two cases.

Case (a). There exists a vertex $y \in B$, which is adjacent to every vertex $x_{i}$ for all $i$, $1 \leq i \leq p-4$.

Let yuzy be a Hamiltonian cycle in $D[B]$.

If $y$ and $x_{0}$ are adjacent then using the observation $\left(^{*}\right)$, it is not difficult to show that either $d^{-}(y, V(C))=0$ or $d^{+}(y, V(C))=0$. Without loss of generality, we assume that $d^{+}(y, V(C))=0$. Then $V(C) \mapsto y$. This together with Claim 1 implies that $A(B \rightarrow V(C))=$ $\emptyset$, which contradicts that $D$ is 2 -strong. We may therefore assume that $y$ and $x_{0}$ are not adjacent. If $x_{1} y \in A(D)$, then $\left\{x_{1}, x_{2}, \ldots, x_{p-4}\right\} \rightarrow y$. Therefore, $A\left(B \rightarrow V(C) \backslash\left\{x_{1}\right\}\right)=\emptyset$. This means that $D\left[V(D) \backslash\left\{x_{1}\right\}\right]$ is not strong, i.e., $D$ is not 2-strong, a contradiction. Now assume that $x_{1} y \notin A(D)$. Then $y x_{1} \in A(D)$ since $y$ and $x_{1}$ are adjacent. Similarly, $x_{p-4} y \in A(D)$. Then by the above observation $(*), d\left(x_{0}, B\right)=0$. Let $x_{k} y \in A(D)$ with $2 \leq k \leq p-4$ and $k$ be the minimum possible. It is not dificult to show that

$$
\left\{x_{k}, x_{k+1}, \ldots, x_{p-4}\right\} \rightarrow y \rightarrow\left\{x_{1}, x_{2}, \ldots, x_{k-1}\right\} .
$$

Assume first that $k \leq p-5$. Then by Claim $1, d^{-}\left(x_{p-4}, B\right)=0$. If $x_{p-4} z \in A(D)$, then $D(p, 2)=\left[x_{p-4} z ; x_{p-4} x_{0} x_{1} \ldots x_{p-5} y u z\right]$, a contradiction. Therefore, $x_{p-4} z \notin A(D)$. Thus, 
$d\left(z,\left\{x_{0}, x_{p-4}\right\}\right)=0$ and $d\left(z,\left\{x_{1}, x_{2}, \ldots, x_{p-5}\right\}\right) \geq p-4$. Again using Lemma $2(\mathrm{i})$ and $(*)$, we obtain that $x_{p-5} z \in A(D)$. Therefore, $x_{p-4} x_{0} x_{1} \ldots x_{p-5} z y$ is a path of length $p-2$ through $x_{0}$ and $x_{p-4} y \in A(D)$, which contradicts Claim 4 .

Assume next that $k=p-4$. Then $y \rightarrow\left\{x_{1}, x_{2}, \ldots, x_{p-5}\right\}$. Hence, if $p-5 \geq 2$, then for the converse digraph of $D$ we have the considered former case. For $4 \leq p \leq 6$, this completes the discussion of Case (a).

Case (b). For every $y \in B$ there exists a vertex $x_{k}$ with $1 \leq k \leq p-4$ such that $y$ and $x_{k}$ are not adjacent.

To complete the proof of Lemma 4 in this case, we first prove the following claims.

Claim 5: $x_{k-1} y \notin A(D)$ or $y x_{k+1} \notin A(D)$.

Proof: Suppose, on the contrary, that $x_{k-1} y \in A(D)$ and $y x_{k+1} \in A(D)$. Note that $d\left(x_{k}\right) \geq p$ since $x_{k} \neq x_{0}$. Using observation $(*)$, we obtain that $d\left(x_{k}, B\right)=0$. Now consider the cycle $R:=x_{0} x_{1} \ldots x_{k-1} y x_{k+1} \ldots x_{p-4} x_{0}$ of length $p-3$ through $x_{0}$. By Claim $1, x_{k}$ cannot be inserted into $R$ (for otherwise we obtain a cycle of length $p-2$ througth $x_{0}$ ). Therefore by Lemma $1, p \leq d\left(x_{k}\right)=d\left(x_{k}, B\right)+d\left(x_{k}, V(R)\right) \leq p-3$, a contradiction.

Claim 6: (i) If $x_{k-1} y \in A(D)$, then $x_{k+1} y \notin A(D)$. (ii) If $y x_{k-1} \in A(D)$, then $y x_{k+1} \notin$ $A(D)$.

Proof: (i) Suppose that the claim is not true. Then $\left\{x_{k-1}, x_{k+1}\right\} \rightarrow y$. By Claim 5, $y x_{k+1} \notin A(D)$. Since $y$ cannot be inserted into the path $C\left[x_{k+1}, x_{k-1}\right]$ and $y x_{k+1} \notin A(D)$, from Lemma 2(ii) it follows that $d(y, V(C))=p-4$.

Assume first that there is a vertex $x_{s} \neq x_{k}$ such that $y$ and $x_{s}$ also are not adjacent. Let $s$ be chosen so that $\left|V\left(C\left[x_{k}, x_{s}\right]\right)\right|$ is the minimum possible. Note that $x_{s} \notin\left\{x_{k-1}, x_{k+1}\right\}$.

Write $P_{1}:=C\left[x_{k+1}, x_{s-1}\right]$ and $P_{2}:=C\left[x_{s+1}, x_{k-1}\right]$. Then

$$
p-4=d(y, V(C))=d\left(y, V\left(P_{1}\right)+d\left(y, V\left(P_{2}\right) \leq\left|V\left(P_{1}\right)\right|+\left|V\left(P_{2}\right)\right|+1=p-4 .\right.\right.
$$

This implies that $d\left(y, V\left(P_{1}\right)\right)=\left|V\left(P_{1}\right)\right|$ and $d\left(y, V\left(P_{2}\right)\right)=\left|V\left(P_{2}\right)\right|+1$. Now using Lemma 2, we obtain $x_{s-1} y \in A(D)$ and $y x_{s+1} \in A(D)$. By Claim $5, x_{s}=x_{0}$ and $d^{-}\left(x_{k+1}, B\right)=$ $d\left(x_{s}, B\right)=0$. Rcall that $z y u z$ is a Hamiltonian cycle in $D[B]$. If $x_{k} z \in A(D)$, then $D(p, 2)=\left[x_{k} z ; C\left[x_{k}, x_{k-1}\right] y u z\right]$, a contradiction. Therefore, $z$ and $x_{k}$ are not adjacent. Now using Lemma $2, d(z) \geq p, d\left(z,\left\{x_{k}, x_{s}\right\}\right)=0$ and the fact that $d^{+}\left(z,\left\{x_{k+1}, x_{k+2}\right\}\right)=0$, we obtain $x_{k+1} z \in A(D)$. Therefore, $C\left[x_{k+1}, x_{k-1}\right] y u z$ is a path of length $p-2$ thruogh $x_{0}$ and $x_{k+1} z \in A(D)$, which contradicts Claim 4 .

Assume next that $y$ is adjacent to every vertex of $V(C) \backslash\left\{x_{k}\right\}$. Then by Claim 1 , $V(C) \backslash\left\{x_{k}\right\} \mapsto y$ since $x_{k+1} y \in A(D)$ and $y x_{k+1} \notin A(D)$. Again using observation (*), we obtain that $A(B \rightarrow V(C))=\emptyset$, which contradicts that $D$ is 2 -strong. This completes the proof of Claim 6(i).

For the proof of Claim 6(ii), it suffices to consider the converse digraph of $D$. Claim 6 is proved.

Claim 7: If $y x_{k-1} \in A(D)$, then $x_{k+1} y \notin A(D)$.

Proof: For a proof by contradiction, suppose that $y x_{k-1} \in A(D)$ and $x_{k+1} y \in A(D)$. Claim 6 implies that $x_{k-1} y \notin A(D)$ and $y x_{k+1} \notin A(D)$. Since $y$ cannot be inserted into $C\left[x_{k+1}, x_{k-1}\right]$, using Lemma $2\left(\right.$ iii), we obtain $d\left(y, V\left(C\left[x_{k+1}, x_{k-1}\right]\right) \leq p-5\right.$, which contradicts that $d(y, V(C)) \geq p-4$. Claim 7 is proved.

Claim 8: (i) The vertices $y$ and $x_{k+1}$ are adjacent; (ii) The vertices $y$ and $x_{k-1}$ are adjacent. Proof: (i) Suppose that the claim is not true, i.e., $d\left(y,\left\{x_{k}, x_{k+1}\right\}\right)=0$. Write $Q:=$ 
$C\left[x_{k+2}, x_{k-1}\right]$. Then $d(y, V(Q))=p-4$. Therefore by Lemma $2, y x_{k+2} \in A(D)$ and $x_{k-1} y \in A(D)$ since $y$ cannot be inserted into $Q$.

Assume first that $x_{k+1} \neq x_{0}$. We know that $d\left(x_{k}\right) \geq p$ and $d\left(x_{k+1}\right) \geq p$. Using obveration $\left(^{*}\right)$, it is not difficult to show that $d\left(x_{k}, B\right)=d\left(x_{k+1}, B\right)=0$. Therefore, $d\left(x_{k}, V(Q)\right) \geq p-2$ and $d\left(x_{k+1}, V(Q)\right) \geq p-2$. These together with Lemmas 2 and 3 imply that the vertices $x_{k}$ and $x_{k+1}$ both can be inserted into $Q$. As a consequence, we obtain a cycle of length $p-2$ through $x_{0}$, which contradicts Claim 1 .

Assume next that $x_{k+1}=x_{0}$. Then $d\left(x_{0}, B\right)=d^{-}\left(x_{k}, B\right)=0$. If $x_{k} z \in A(D)$, then $D(p, 2)=\left[x_{k} z ; C\left[x_{k}, x_{k-1}\right] y u z\right]$, a contradiction. If $x_{k} u \in A(D)$, then $C\left[x_{k}, x_{k-1}\right] y z$ is a path of length $p-2$ through $x_{0}$ and $x_{k} u \in A(D)$, which contradicts Claim 4. We may therefore assume that $d\left(z,\left\{x_{k}, x_{k+1}\right\}\right)=d\left(u,\left\{x_{k}, x_{k+1}\right\}\right)=0$. Therefore, $d(z, V(Q))=p-4$, $z x_{k+2}$ and $x_{k-1} z \in A(D)$. Now using Claims 1 and 5, we obtain that there is a vertex $x_{s}$ such that $\{y, z\} \rightarrow V\left(C\left[x_{k+2}, x_{s}\right]\right)$ and $V\left(C\left[x_{s}, x_{k-1}\right]\right) \rightarrow\{y, z\}$. Whitout loss of generality, assume that $\left|V\left(C\left[x_{k+2}, x_{s}\right]\right)\right| \geq 2$ (for otherwise we consider the converse digraph of $D$ ). Then $D(p, 2)=\left[y x_{k+2} ; y u z C\left[x_{k+3}, x_{k+2}\right]\right]$, a contradiction. This contradiction completes the proof of Claime 8(i). By the same arguments one can prove Claim 8(ii). Claim 8 is proved.

Now we return to the proof of the lemma. From Claim 8 it follows that $y$ is adjacent to $x_{k-1}$ and $x_{k+1}$. Therefore, only the following cases are possible: (i) $x_{k-1} y$ and $y x_{k+1} y \in A(D)$, (ii) $\left\{x_{k-1}, x_{k+1}\right\} \rightarrow y$, (iii) $y \rightarrow\left\{x_{k-1}, x_{k+1}\right\}$, (iv) $x_{k+1} y$ and $y x_{k-1} \in A(D)$. On the other hand, Claims 5, 6 and 7 imply that none of these cases holds. This contradiction completes the discussion of Case (b). Lemma 4 is proved.

Now we are ready to complete the proof of the theorem in Case 2. Since $D$ is not Hamiltonian, by Corollary $1, D$ contains a cycle of length $p-1$. Let $R:=x_{1} x_{2} \ldots x_{p-1} x_{1}$ be a cycle of length $p-1$ in $D$. Then by Claim 1 and Lemma 4 , we know $x_{0} \notin V(R)$ and for every $i, j, 1 \leq i, j \leq p-1$ the following hold:

$$
\begin{aligned}
& d^{-}\left(x_{0},\left\{x_{i}\right\}\right)+d^{+}\left(x_{0},\left\{x_{i+1}, x_{i+2}, x_{i+3}, x_{i+4}\right\}\right) \leq 1, \\
& d^{+}\left(x_{0},\left\{x_{j}\right\}\right)+d^{-}\left(x_{0},\left\{x_{j-1}, x_{j-2}, x_{j-3}, x_{j-4}\right\}\right) \leq 1 .
\end{aligned}
$$

Therefore, $d^{-}\left(x_{0}\right)+4 d^{+}\left(x_{0}\right) \leq p-1$ and $4 d^{-}\left(x_{0}\right)+d^{+}\left(x_{0}\right) \leq p-1$. These mean that $5 d\left(x_{0}\right) \leq 2 p-2$, i.e., $d\left(x_{0}\right) \leq 2(p-1) / 5$, which contradicts that $d\left(x_{0}\right)>2(p-1) / 5$. The theorem is proved.

Corollary 2: (Benhocine [5]). Every strong digraph D of order $p \geq 3$ and with minimum degree at least $p$ contains $D(p, 2)$.

Proof: By the famous theorem of Ghoula-Houri, $D$ is Hamiltonian. Therefore, from the proof of Theorem 6 in Case (a), it follows that $D$ contains a Hamiltonian bypass.

Perhaps the following proposition will be useful for Conjecture 1 (see, in section Conclusion).

Proposition 1: Let $D$ be a non-Hamiltonian 2-strong digraph of order $p \geq 3$. Suppose that $d(x) \geq p$ for every vertex $x \in V(D) \backslash\left\{x_{0}\right\}$, where $x_{0}$ is a vertex of $D$. If $P=x_{1} x_{2} \ldots x_{p-2}$ is an $\left(x_{1}, x_{p-2}\right)$-path of length $p-3$ through $x_{0}$ in $D$, then $x_{1} x_{p-2} \notin A(D)$. 
Proof: For a proof by contradiction, suppose that $x_{1} x_{p-2} \in A(D)$. Write $V(D) \backslash V(P)=$ $\left\{y_{1}, y_{2}\right\}$. We know that $d\left(y_{1}\right) \geq p$ and $d\left(y_{2}\right) \geq p$ since $x_{0} \in V(P)$. From Claim 4 it follows that $y_{i}$ cannot be inserted into $P$. On the other hand, since $D$ contains no cycle of length $p-1$ through $x_{0}$, we have that $y_{i} x_{1} \notin A(D)$ or $x_{p-2} y_{i} \notin A(D)$. Now using Lemma 2(ii), we obtain $d\left(y_{i}, V(P)\right)=p-2$ and $y_{1} y_{2}, y_{2} y_{1} \in A(D)$. Without loss of generality, assume that $y_{1} x_{1} \notin A(D)$. Then by Lemma 2(ii), $x_{p-2} y_{1} \in A(D)$. Since $D$ is not Hamiltonian and contains no cycle of length $p-1$ through $x_{0}$, it follows that $d^{-}\left(x_{1},\left\{y_{1}, y_{2}\right\}\right)=$ 0 . Then $x_{p-2} y_{2} \in A(D)$. If $x_{1} y_{1} \in A(D)$ (or $x_{1} y_{2} \in A(D)$ ), then it is not difficult to show that $D(p, 2)=\left[x_{1} y_{1} ; x_{1} x_{2} \ldots x_{p-2} y_{2} y_{1}\right]$ (or $\left.D(p, 2)=\left[x_{1} y_{2} ; x_{1} x_{2} \ldots x_{p-2} y_{1} y_{2}\right]\right)$ is in $D$, a contradiction. Therefore, $d^{+}\left(x_{1},\left\{y_{1}, y_{2}\right\}\right)=0$. Thus, $d\left(x_{1},\left\{y_{1}, y_{2}\right\}\right)=0$. This together with Lemma 2 and $d\left(y_{i}, V(P)\right)=p-2$ implies that $\left\{y_{1}, y_{2}\right\} \rightarrow x_{2}$. Then by Claim $1, x_{1}=x_{0}$ since $x_{2} x_{3} \ldots x_{p-2} y_{1} y_{2} x_{2}$ is a cycle of length $p-1$.

Write $Q:=x_{2} x_{3} \ldots x_{p-2}$. Then $|V(Q)|=p-3, d\left(y_{1}, V(Q)\right) \geq p-2$ and $d\left(y_{2}, V(Q)\right) \geq$ $p-2$. Since $x_{0} \rightarrow\left\{x_{2}, x_{p-2}\right\}$, by Claim 4 we have that neither $y_{1}$ nor $y_{2}$ can be inserted into $Q$. Then by Lemma 2(iii), we obtain that $d\left(y_{1}, V(Q)\right)=d\left(y_{2}, V(Q)\right)=p-2$ and the arcs $y_{2} y_{1}, x_{p-2} y_{2}, y_{1} x_{2}$ are in $A(D)$.

We claim that the vertex $y_{1}\left(y_{2}\right)$ is adjacent to each vertex of $V(Q)$.

Assume that this is not the case. Let $d\left(y_{1},\left\{x_{i}\right\}\right)=0$, where $3 \leq i \leq p-3$. From Lemma 2(iii), $d\left(y_{1}, V(Q)\right)=p-2$ and the fact that the vertex $y_{1}$ cannot be inserted into $Q$ it follows that $x_{i-1} y_{1}, y_{1} x_{i+1} \in A(D)$. Since $y_{2} y_{1}, y_{1} y_{2} \in A(D)$, it is easy to see that $d\left(y_{2},\left\{x_{i}\right\}\right)=0$ and $x_{i-1} y_{2}, y_{2} x_{i+1} \in A(D)$. They imply that the vertex $x_{i}$ can be inserted neither into $S:=x_{2} \ldots x_{i-1}$ nor into $T:=x_{i+1} \ldots x_{p-2}$. Then it is easy to see that $d\left(x_{i},\left\{x_{0}\right\}\right)=2$ and

$$
p-2 \leq d\left(x_{i}, V(S)\right)+d\left(x_{i}, V(T)\right) \leq|V(S)|+|V(T)|+2=p-2 .
$$

Therefore, $d\left(x_{i}, V(S)\right)=|V(S)|+1$ and $d\left(x_{i}, V(T)\right)=|V(T)|+1$. Again using Lemma 2, we obtain $x_{p-2} x_{i}$ and $x_{i} x_{2} \in A(D)$. Hence, $x_{0} x_{i} x_{2} \ldots x_{i-1} y_{1} y_{2} x_{i+1} \ldots x_{p-2}$ is an $\left(x_{0}, x_{p-2}\right)$ Hamiltonian path, a contradiction as $x_{0} x_{p-2} \in A(D)$. This proves that $y_{1}\left(y_{2}\right)$ is adjacent to every vertex in $V(Q)$. Therefore, there is an integer $l, 2 \leq l \leq p-2$, such that

$$
\left\{x_{l}, x_{l+1}, \ldots, x_{p-2}\right\} \rightarrow\left\{y_{1}, y_{2}\right\} \rightarrow\left\{x_{2}, x_{3}, \ldots, x_{l}\right\}
$$

If $3 \leq l \leq p-3$, then by (2), $x_{0} x_{p-2} y_{1} x_{3} \ldots x_{p-3} y_{2} x_{2}$ is an $\left(x_{0}, x_{2}\right)$-Hamiltonian path, which is a contradiction as $x_{0} x_{2} \in A(D)$. If $l=2$ or $l=p-2$, then $A\left(\left\{y_{1}, y_{2}\right\} \rightarrow V(D) \backslash\left\{x_{2}\right\}\right)=\emptyset$ or $A\left(V(D) \backslash\left\{x_{p-2}\right\} \rightarrow\left\{y_{1}, y_{2}\right\}\right)=\emptyset$ when $l=2$ or $l=p-2$, respectively. This means that $D$ is not 2-strong, a contradiction. Proposition 1 is proved.

\section{Conclusion}

In the current article, we examined the existence of a Hamiltonian bypass in 2-strong digraphs of order $p$, in which $p-1$ vertices have degrees at least $p$. We proved that if such digraphs are Hamiltohian or have the minimal degree more than $2(p-1) / 5$, then such digraphs contain a Hamiltonian bypass.

If we consider the digraph $H(n)$ (by Remark $1, H(n)$ is 2-strong, $d\left(x_{0}\right)=4$ and is not Hamiltonian), then we see that $D(n, 2)=\left[y_{1} x_{1} ; y_{1} y_{2} y_{3} x_{2} x_{3} \ldots x_{n-4} x_{0} x_{1}\right]$ is a Hamiltonian bypass. By the above arguments, we believe that the following conjecture is true.

Conjecture 1: Let $D$ be a 2-strong digraph of order $p$. If $p-1$ vertices in $V(D)$ have degrees at least $p$, then $D$ contains a Hamiltonian bypass. 


\section{Acknowledgements}

The author would like to thank the referees of the paper for careful reading and many helpful remarks.

\section{References}

[1] J. Bang-Jensen, G. Gutin, Digraphs: Theory, Algorithms and Applications, Springer, 2001.

[2] J.-C. Bermond and C. Thomassen, "Cycles in Digraphs-A Survey", Journal of Graph Theory, vol. 5, pp. 1-43, 1981.

[3] D. Kühn and D. Osthus, "A survey on Hamilton cycles in directed graphs", European Journal of Combinatorics, vol. 33, pp. 750-766, 2012.

[4] A. Benhocine and A.P. Wojda, "Bypasses in Digraphs", Ars Combinatoria, vol. 16, pp. 85-94, 1983.

[5] A. Benhocine, "On the existence of a specified cycle in digraphs with constraints on degrees", Journal of Graph Theory, vol. 8, pp. 101-107, 1984.

[6] S.Kh. Darbinyan, "On Hamiltonian bypasses in digraphs satisfying Meyniel-like condition", Transactions of IIAP of NAS RA, Mathematical Problems of Computer Science, vol. 20, pp. 7-19, 1998.

[7] S.Kh. Darbinyan, "On the specified cycles in oriented graphs", Akademy Nauk Armyan. SSR Dokllady, vol. 84, no. 2, pp. 51-55, 1987 (in Russian).

[8] S.Kh. Darbinyan and I.A. Karapetyan, "On Hamiltonian bypasses in one class of Hamiltonian digraphs", Mathematical Problems of Computer Science, vol. 41, pp. 23-37, 2014.

[9] S.Kh. Darbinyan, "On Hamiltonian bypasses in digraphs with the condition of Y. Manoussakis", "2015 Computer Science and Information Technologies (CSIT), Yerevan, doi:10.1109/CSITechnol.2015.7358250, pp. 53-63, 2015.

[10] S.Kh. "On Hmiltonian and strongly Hamiltonconnected digraphs", Akademy Nauk Armyan. SSR Doklady, vol. 91, no. 1, pp. 3-6, 1990.(arXiv.1801.05166v1).

[11] S.Kh. Darbinyan, "A sufficient condition for a digraph to be Hamiltonian", Akademy Nauk Armyan. SSR Doklady,(in Russian), vol. 91, no. 2, pp. 57-59, 1990.

[12] R. Häggkvist and C. Thomassen, "On pancyclic digraphs", Journal Combinatorial Theory ser. B, vol. 20, pp. 20-40, 1976.

[13] J.A. Bondy and C. Thomassen, "A short proof of Meyniel's theorem", Discrete Mathematics, vol. 19, pp. 195-197, 1977.

[14] J. Bang-Jensen, G. Gutin and H. Li, "Sufficient conditions for a digraph to be Hamiltonian", Journal of Graph Theory, vol. 22, no. 2, pp. 181-187, 1996.

Submitted 03.08.2020, accepted 01.12.2020. 


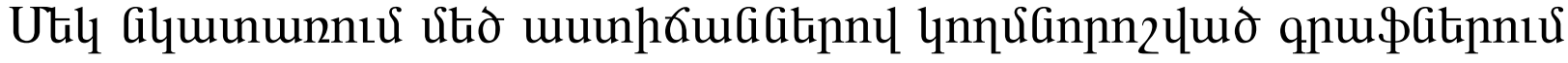

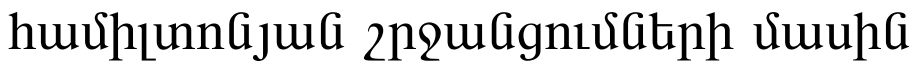

\author{
Uuulthl k. R-upphajua

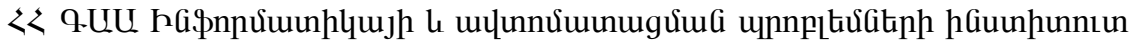 \\ e-mail: samdarbin@iiap.sci.am
}

\section{Uựnnนnนu}

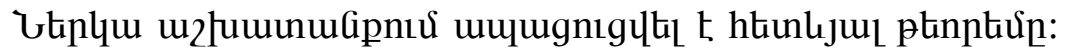

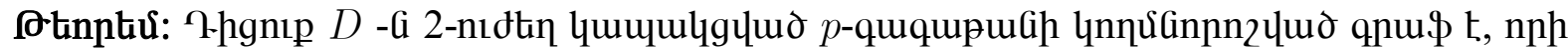

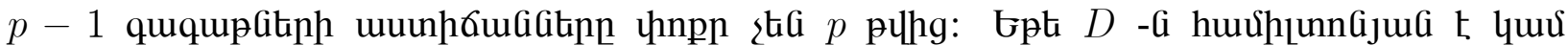

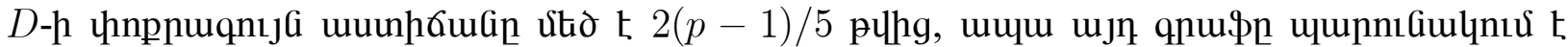
hứhıunnajuGi 2nguGignıu:

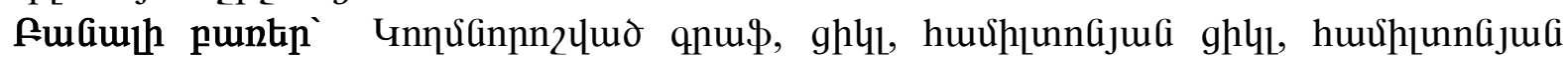
2nquagntu:

\section{ОАна заметка о гамильтоновых обходах в орграфах с большими степеньями}

\author{
Самвел Х. Аарбинян \\ Институт проблем информатики и автоматизации НАН РА \\ e-mail: samdarbin@iiap.sci.am
}

\begin{abstract}
Аннотация
В настоящей работе доказана следующая теорема:

Теорема: Пусть $D$ есть 2-сильно связный $p$-вершинный орграф, в котором $p-1$ вершины имеют степень не меньше чем $p$. Если $D$ гамильтонов или имеет минимальную степень больше чем $2(p-1) / 5$, то $D$ содержит гамильтонов обход.

Ключевые слова: Орграф, цикл, гамильтонов цикл, гамильтонов обход.
\end{abstract}

